\title{
Hierarchical control of aerial manipulation vehicle
}

Somasundar Kannan, Souad Bezzaoucha, Serket Quintanar Guzman, Jan Dentler, Miguel A. Olivares-Mendez, and Holger Voos

Citation: 1798, 020069 (2017); doi: 10.1063/1.4972661

View online: http://dx.doi.org/10.1063/1.4972661

View Table of Contents: http://aip.scitation.org/toc/apc/1798/1

Published by the American Institute of Physics 


\title{
Hierarchical Control of Aerial Manipulation Vehicle
}

\author{
Somasundar Kannan ${ }^{1, a)}$, Souad Bezzaoucha ${ }^{1}$, Serket Quintanar Guzman ${ }^{1}$, Jan \\ Dentler $^{1}$, Miguel A. Olivares-Mendez ${ }^{1}$ and Holger $\operatorname{Voos}^{1}$ \\ ${ }^{1}$ SnT- University of Luxembourg, 6 rue Richard Coudenhove-Kalergi, Kirchberg, L-1359 Luxembourg \\ a) Corresponding author: somasundar.kannan@uni.lu
}

\begin{abstract}
Hierarchical Control of the Aerial Manipulator is treated here. The modelling aspect of the highly coupled Aerial Vehicle which includes Quadrotor and manipulator is discussed. The control design to perform tasks in operational space is addressed along with stability discussion. The simulation studies are successfully performed to validate the design methodology.
\end{abstract}

\section{INTRODUCTION}

The design and development of Aerial Manipulators have increased the applications of Unmanned Aerial Vehicles (UAVs). With these developments the new applications of aerial vehicles include grasping, manipulation, transportation etc. This advancement has further lead to new challenges.

In [1] and [2] cartesian impedance control and redundancy is discussed. In [3] outer loop adaptive control was used to control the Aerial Manipulator and in [4] vision sensors were used for control simulations.

In [5] a hybrid visual servoing was used to control an aerial vehicle with redundant robotic arm. In [6] an adaptive sliding mode control was implemented on an Aerial Manipulator. In [7] and [8] operational space control was discussed on an aerial vehicle with redundant manipulator. In [9] operational space control was discussed for an Aerial Vehicle equipped with a non-redundant manipulator

In current paper we discuss the hierarchical control structure of an Aerial Vehicle attached with 2 degree of freedom manipulator. Compared with [9] the key contribution of current paper includes the stability discussions of the hierarchical control structure.The paper is structured in the following way. First the modelling is discussed followed by control and stability analysis. Finally simulation studies are performed for validation

\section{MODELLING}

The kinematics and dynamics of the Aerial Manipulation Vehicle is briefly discussed here based on [7]. The CAD design of the Aerial Manipulation Vehicle can be seen in Figure-1 while the 2 degree of freedom manipulator can be seen in Figure-2.

\section{Kinematics}

The vehicle body frame fixed to the center of mass of the quadrotor is given by $\mathcal{B}$. The position of $\mathcal{B}$ in the world

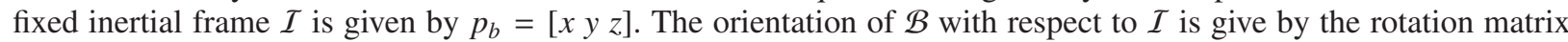
$R_{b}\left(\varphi_{b}\right)$. Consider $\varphi_{b}=[\psi \theta \phi]$ as the Yaw-Pitch-Roll angles. The frame attached to the end-effector of the manipulator is defined by $\mathcal{E}$. The position of $\mathcal{E}$ with respect to $\mathcal{I}$ is given by [7]

$$
p_{e}=p_{b}+R_{b} p_{e b}^{b},
$$

where $p_{e b}^{b}$ gives the position of $\mathcal{E}$ with respect to $\mathcal{B}$. The orientation of $\mathcal{E}$ can be described by the rotation matrix [7]

$$
R_{e}=R_{b} R_{e}^{b}
$$


where $R_{e}^{b}$ gives the orientation of $\mathcal{E}$ with respect to $\mathcal{B}$. The differential kinematics expression $v_{e}$ required for the inverse kinematic algorithm can be given as [7]

$$
v_{e}=J_{\gamma}\left(\sigma_{b}, \gamma\right) \dot{\gamma}+J_{\sigma}\left(\sigma_{b}, \gamma\right) \dot{\sigma}_{b}
$$

where $\gamma=\left[\begin{array}{ll}\mu_{b}^{T} & q^{T}\end{array}\right]$ is the vector of controlled variables, $J_{\eta}$ is composed by first 4 columns of $J_{b} T_{A}\left(\varphi_{b}\right), J_{\sigma}$ is composed by the last 2 columns of $J_{b} T_{A}\left(\varphi_{b}\right)$, and $J_{\gamma}=\left[\begin{array}{ll}J_{\eta} & J_{e b}\end{array}\right]$. We have

$$
J_{b}=\left[\begin{array}{cc}
I_{3} & -S\left(R_{b} p_{e b}^{b}\right) \\
O_{3} & I_{3}
\end{array}\right], J_{e b}=\left[\begin{array}{ll}
R_{b} & O_{3} \\
O_{3} & R_{b}
\end{array}\right] J_{e b}^{b},
$$

where $J_{e b}^{b}$ is the manipulator Jacobian and $S(\cdot)$ is a skew symmetric matrix operator. We further need

$$
T_{A}\left(\varphi_{b}\right)=\left[\begin{array}{cc}
I_{3} & O_{3} \\
O_{3} & T\left(\varphi_{b}\right)
\end{array}\right]
$$

where $T\left(\varphi_{b}\right)$ can be given by

$$
T\left(\varphi_{b}\right)=\left[\begin{array}{ccc}
0 & -s \psi & c \psi c \theta \\
0 & c \psi & s \psi c \theta \\
1 & 0 & -s \theta
\end{array}\right]
$$

We further have $q$ as the joint coordinates of the manipulator and

$$
\mu_{b}=\left[\begin{array}{c}
p_{b} \\
\psi
\end{array}\right], \sigma_{b}=\left[\begin{array}{l}
\theta \\
\phi
\end{array}\right] .
$$

The detailed discussion of the above kinematic discussion can be obtained from [7].
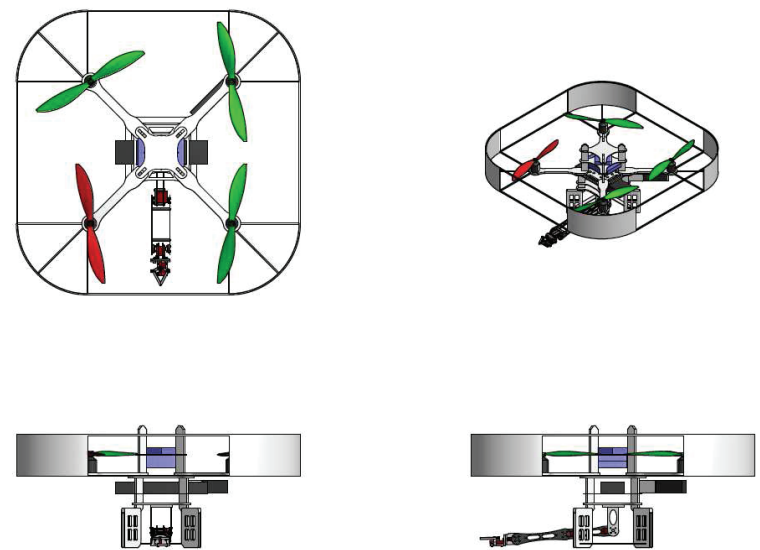

FIGURE 1. Aerial Manipulation Vehicle.

\section{Dynamics}

The dynamic model of the aerial manipulation vehicle can be given as [7]

$$
\mathbf{A}(\chi) \ddot{\chi}+\mathbf{B}(\chi, \dot{\chi}) \dot{\chi}+g(\chi)=u
$$

where $\chi=\left[\begin{array}{ll}x_{b}^{T} & q^{T}\end{array}\right], \mathbf{A}$ is the inertia matrix, $\mathbf{B}$ is composed of the coriolis and centrifugal terms, $g$ is the gravity term and $u$ is the input vector [7]

$$
u=\left[\begin{array}{l}
u_{f} \\
u_{v} \\
u_{\tau}
\end{array}\right]=\left[\begin{array}{c}
R_{b}(\varphi) f_{b}^{b} \\
T^{T}\left(\varphi_{b}\right) R\left(\varphi_{b}\right) v_{b}^{b} \\
\tau
\end{array}\right]
$$

where $\tau$ are the torques applied to the manipulator joint, $f_{b}^{b}$ and $v_{b}^{b}$ are the forces and moments on the quadrotor expressed in $\mathcal{B}$. 


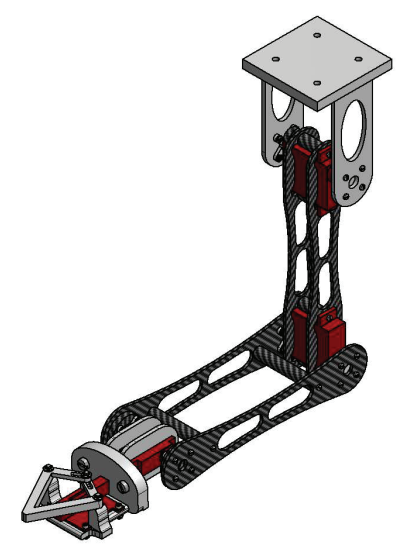

FIGURE 2. 2 DOF Robotic Arm.

\section{CONTROL}

The hierarchical control scheme can be seen in Figure-3. The first layer consist of the Closed Loop Inverse Kinematics followed by position and attitude control for the quadrotor and then the joint control for the manipulator. Here we will briefly discuss the control problem.

\section{Closed Loop Inverse Kinematics}

The Closed Loop Inverse Kinematics algorithm is necessary to generate the reference trajectories to the position, attitude and joint control loops for the quadrotor and manipulator respectively. The closed loop inverse kinematics is given by [7],[9]

$$
\begin{aligned}
\dot{\gamma}_{r}= & J_{\gamma}^{\dagger}\left(\sigma_{b}, \gamma_{r}\right) T_{A}\left(\varphi_{e, r}\right)\left(\dot{x}_{e, d}+K e\right) \\
& -J_{\gamma}^{\dagger}\left(\sigma_{b}, \gamma_{r}\right) J_{\sigma}\left(\sigma_{b}, \gamma_{r}\right) \dot{\sigma}_{b}
\end{aligned}
$$

where $J_{\gamma}^{\dagger}$ is the pseudoinverse of $J_{\gamma}, K$ is the symmetric positive definite gain and $e=x_{e, d}-x_{e, r}$ is the end-effector position and attitude error.

\section{Closed Loop Control Structure}

The hierarchical control structure of the quadrotor can be seen in Figure-3. The quadrotor control consists of two loops including attitude and position. The roll and pitch controller can be given by [9]

$$
U_{r p}(t)=K_{p}\left[\varepsilon_{r p}(t)-K_{d} \dot{\Theta}_{r p}(t)\right]
$$

where $\varepsilon_{r p}=\Theta_{r p}^{*}-\Theta_{r p}$. Here $\Theta_{r p}^{*}$ is the roll and pitch demand and finally $U_{r p}$ gives us pitch $\left(\tau_{y}\right)$ and roll torque $\left(\tau_{x}\right)$. A PID control for the yaw control loop can be given by [9]

$$
U_{\text {yaw }}(t)=K_{p}\left[\varepsilon_{\text {yaw }}(t)+K_{i} \int_{0}^{t} \varepsilon_{\text {yaw }}(\delta) d \delta-K_{d} \dot{\Omega}_{z}(t)\right]
$$

where $\varepsilon_{\text {yaw }}=\psi^{*}-\psi$ is the error in the yaw channel and yaw demand is given by $\psi^{*}$.

The $x, y$ position control loop uses a PID type controller. The output of this controller generates the pitch and roll demand [9]

$$
\begin{array}{r}
\Theta_{r p}^{*}(t)=K_{p}\left[\varepsilon_{x y}(t)+K_{i} \int_{0}^{t} \varepsilon_{x y}(\delta) d \delta\right. \\
-K_{d} v_{x y}(t) \\
\left.-K_{d d} \dot{v}_{x y}(t)\right]
\end{array}
$$


where $\varepsilon_{x y}=\xi_{x y}^{*}-\xi_{x y}$ is the $x, y$ - position error and $v_{x y}$ is the position rate and $\dot{v}_{x y}$ is the respective acceleration. For the $z$ - axis control a PID controller is used [9]

$$
T(t)=K_{p}\left[\varepsilon_{z}(t)+K_{i} \int_{0}^{t} \varepsilon_{z}(\delta) d \delta-K_{d} v_{z}(t)-K_{d d} \dot{v}_{z}(t)\right]
$$

where $\varepsilon_{z}=\xi_{z}^{*}-\xi_{z}$ is the $z$ position error and $v_{z}$ is the $z$ position rate and $\dot{v}_{z}$ is the acceleration. A PD type of control is used for the manipulator control [9]

$$
U_{m}(t)=K_{p}\left[K_{p 1} \tilde{q}(t)-K_{d} \dot{q}(t)\right]
$$

where $\tilde{q}$ is a joint angle error.

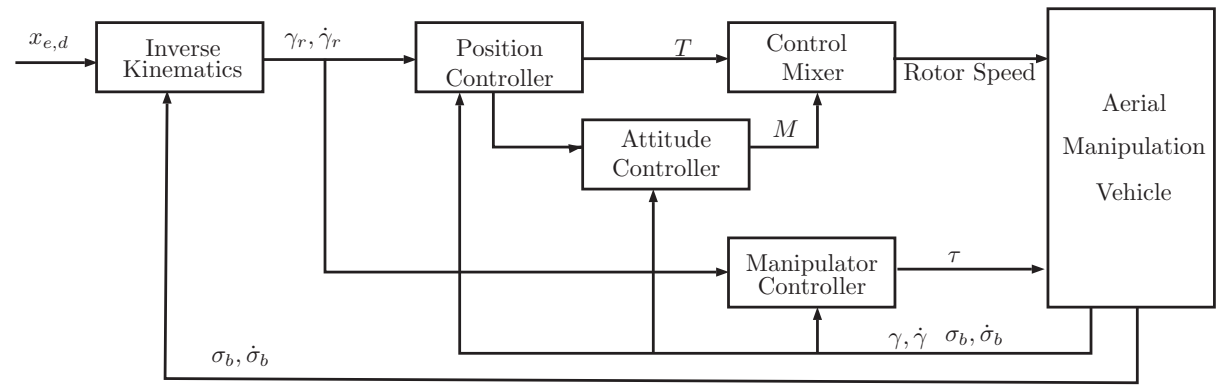

FIGURE 3. Aerial Manipulation Vehicle: Control Structure [9].

\section{Stability Discussion}

In the current section we will briefly discuss the stability of the closed loop system. But before we consider a more basic result. Let an autonomous system with disturbance be described as below [10]:

$$
\dot{x}=A x+U
$$

where $A$ is assumed to a Hurwitz matrix and $U$ can be considered as disturbance. With $A$ being Hurwitz we have the Lyapunov equation $A^{T} P+P A=-Q$ with $Q$ positive definite. Let us choose the following Lyapunov function for the system $V=x^{T} P x$ with $P$ symmetric positive definite. The time derivative of the Lyapunov function along the system trajectory gives us [10],[11]

$$
\begin{aligned}
\dot{V} & =-x^{T} Q x+2 x^{T} P U \\
& \leq-\lambda_{\min }(Q)\|x\|_{2}^{2}+2 \lambda_{\max }(P)\|x\|_{2}\|U\|_{2} \\
& \leq-\lambda_{\min }(Q)\|x\|_{2}\left(\|x\|_{2}-2\|U\| \lambda_{\max }(P)\right)
\end{aligned}
$$

The stability of the system is then ensured if

$$
\|x\|_{2} \geq 2\|U\|_{2} \lambda_{\max }(P)
$$

which implies that the disturbance control input norm is bounded [10], [11]

$$
\|U\|_{2} \leq \frac{1}{2 \lambda_{\max }(P)}\|x\|_{2}
$$

where $\lambda_{\min }$ and $\lambda_{\max }$ are minimum and maximum Eigen values of the respective matrices and $\|\cdot\|_{2}$ is the corresponding 2-norm. 
Now considering the stability discussion of our quadrotor control problem. The inner loop attitude control with different PID controllers can be considered as the system in equation (16). Now considering $V_{a}=x_{a}^{T} P x_{a}$ as the Lyapunov function for different attitude loops such as yaw, pitch and roll, stability can be attained if we satisfy equation (21). Similarly by choosing $V_{p}=x_{p}^{T} P x_{p}$ the position control loop can be stabilized given equation (21) is satisfied.

For the inverse kinematics loop choose the function $e=x_{d}-x_{e}$ as the error between desired and actual endeffector position. According to the discussion in [12] by choosing $V_{e}=e^{T} K e$ the closed loop inverse kinematics algorithm can be rendered asymptotically stable with $K$ positive definite. Finally similar to the discussion in [8] under the assumption that perfect tracking of desired values is obtained and by choosing the Lyapunov function

$$
V_{c l}=V_{e}+V_{p}+V_{a}
$$

for the complete system, $\dot{V}_{c l}$ can be rendered negative definite from negative definite property of $\dot{V}_{e}, \dot{V}_{p}$ and $\dot{V}_{a}$ to attain asymptotic stability of the complete system.

\section{SIMULATION}

In the current section we will briefly discuss the application of the above discussed control methods to our considered benchmark Aerial Manipulator. The benchmark simulation system considered here is an Asctec Pelican Quadrotor which is fixed with a custom made 2-Degree of Freedom manipulator of mass $110 \mathrm{~g}$. The Asctec Pelican Quadrotor has a mass of $2 \mathrm{Kg}$ and Inertia $I=\operatorname{diag}\left[\begin{array}{lll}1.24 & 1.24 & 2.48\end{array}\right]$ which has been obtained from [7]. The design and model of the Aerial manipulator and the manipulator can be seen in Figure-1 and Figure-2.

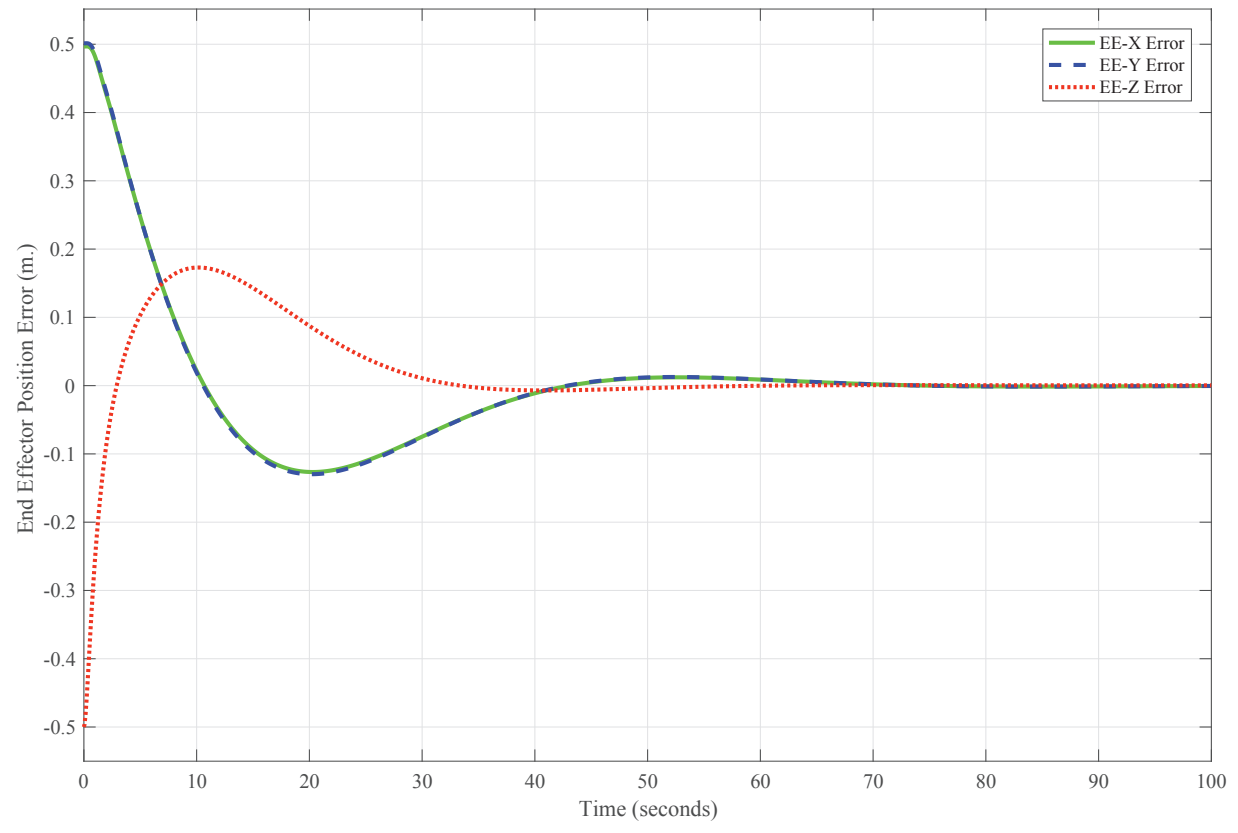

FIGURE 4. End Effector Position Control Error.

The operational space control is tested here on the given Aerial Manipulation Vehicle (AMV). The task here is that the AMV starts from hover condition with quadrotor position starting at $\{0+0.27587,0,2-0.1184\}$ and manipulator end-effector position from $[0,0]$ has to reach $\{0.5+0.27587,0.5,1.5-0.1184\}$. In-order to perform the simulation a single set of control parameters are used. The pitch and roll control gains $K_{p}=5, K_{d}=5$ are used while for the yaw control the gains are $K_{p}=-3, K_{i}=0.001, K d=0.01$. The outer loop position control gains are $K_{p}=$ $[10,-10], K_{i}=[0.1,0.1], K_{d}=[7,7], K_{d d}=[1,1]$ for $x-y$ position control and $K_{p}=10, K_{i}=0.1, K_{d}=5, K_{d d}=1$ are the gains used for $z$ - axis. For the manipulator control the gains used are $K_{p}=1, K_{p 1}=50, K_{d}=0.1$ for each of the joint and the gains for inverse kinematics loop are $K=[1,1,1,1,1,1]$. The gains chosen here are obtained from 


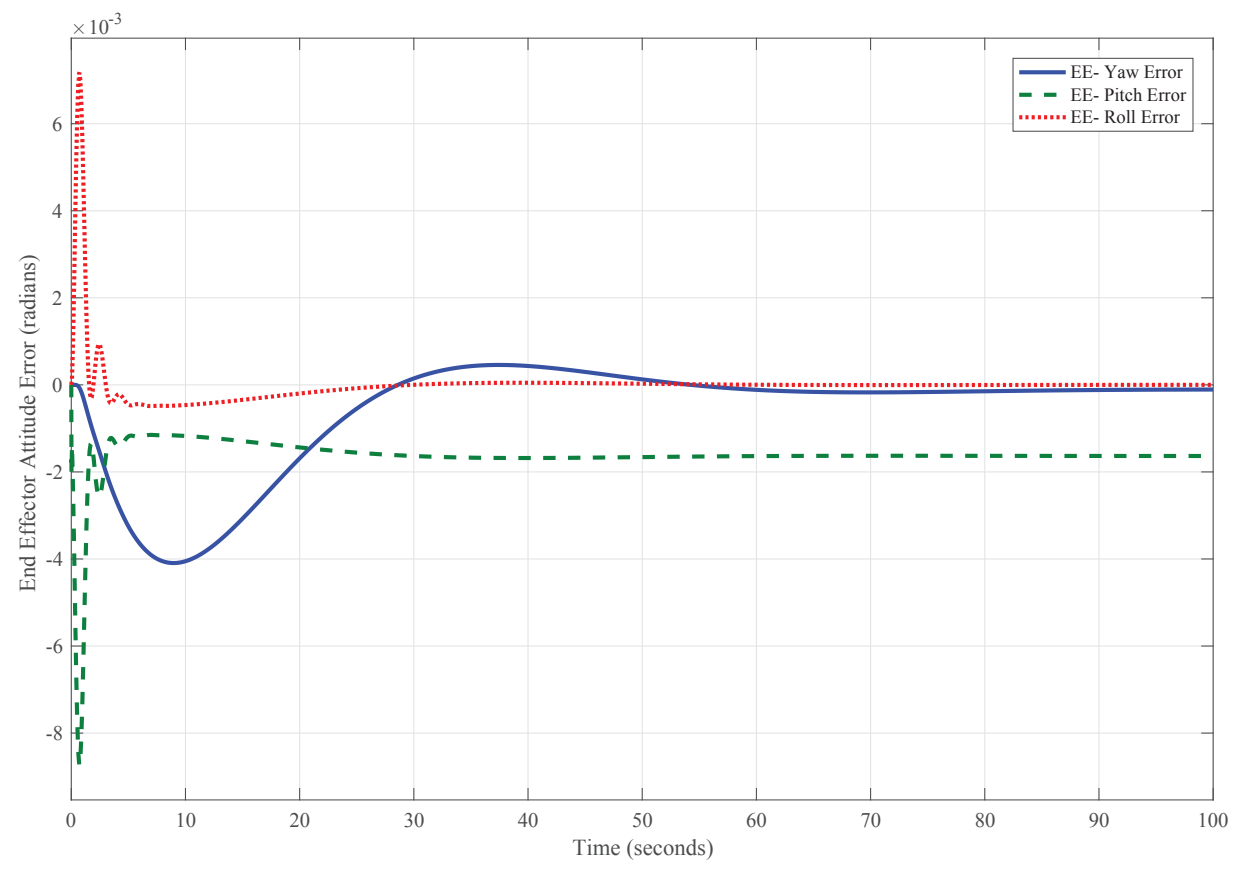

FIGURE 5. End Effector Attitude Control Error.

trial and error procedure. The simulation results can be seen in Figure-4 and Figure-5. In Figure-4 we can see position error of the end-effector while in Figure-5 we can see the attitude error of the end-effector. From the results we can observe that the tracking error asymptotically converges to zero.

\section{CONCLUSION}

In the current paper we discussed the hierarchical control problem of the Aerial Manipulation Vehicle in the operational space. The nonlinear modelling aspect was discussed followed by control design. The stability properties of the hierarchical control system was also addressed and finally simulation was performed to validate the methodology. The future perspective of the current research include experimental validation of the proposed methodology.

\section{REFERENCES}

[1] V. Lipiello and F. Ruggiero, "Cartesian impedence control of a uav with a robotic arm," in 10th IFAC Symposium on Robot Control (2012).

[2] V. Lipiello and F. Ruggiero, "Exploiting redundancy in cartesian impedance control of UAVs equipped with a robotic arm," in 2012 IEEE/RSJ International Conference on Intelligent Robots and Systems (2012), pp. 3768-3773.

[3] S. Kannan, M. Alma, M. A. Olivares-Mendez, and H. Voos, "Adaptive control of aerial manipulation vehicle," in Control System, Computing and Engineering (ICCSCE), 2014 IEEE International Conference on (2014), pp. 273-278.

[4] S. Kannan, M. A. Olivares-Mendez, and H. Voos, "Modeling and control of aerial manipulation vehicle with visual sensor," in 2nd IFAC Workshop on Research, Education and Development of Unmanned Aerial Systems, Vol. 2 (2013).

[5] V. Lippiello, J. Cacace, A. Santamaria-Navarro, J. Andrade-Cetto, M. . Trujillo, Y. R. Esteves, and A. Viguria, IEEE Robotics and Automation Letters 1, 259-266Jan (2016).

[6] S. Kim, S. Choi, and H. J. Kim, “Aerial manipulation using a quadrotor with a two dof robotic arm," in 2013 IEEE/RSJ International Conference on Intelligent Robots and Systems (2013), pp. 4990-4995. 
[7] G. Arleo, F. Caccavale, G. Muscio, and F. Pierri, "Control of quadrotor aerial vehicles equipped with a robotic arm," in Control Automation (MED), 2013 21st Mediterranean Conference on (2013), pp. 11741180.

[8] F. Caccavale, G. Giglio, G. Muscio, and F. Pierri, "Adaptive control for uavs equipped with a robotic arm," in 19th IFAC World COngress, Vol. 19 (2014), pp. 11049-11054.

[9] S. Kannan, S. Q. Guzman, J. Dentler, M. A. Olivares-Mendez, and H. Voos, "Control of aerial manipulation vehicle in operational space," in IEEE International Conference on Electronics, Computers and Artificial Intelligence (ECAI-2016) (2016).

[10] P. N. Kohler and D. V. Dimarogonas, "On topological conditions to maintain leader-follower connectivity in double-integrator multi-agent systems," in 2016 24th Mediterranean Conference on Control and Automation (MED) (2016), pp. 767-772.

[11] W. M. Haddad and J. Chellaboina, Nonlinear dynamical systesm and control: A lyapunov-based approach, 2008.

[12] B. Siciliano, L. Sciavicco, L. Villani, and G. Oriolo, Robotics: modelling, planning and control, 2010. 